\title{
Observation of low-temperature annealing of a primary defect in gallium nitride
}

\author{
Matthias Schmidt, P. Johan Janse van Rensburg, Hannes de Meyer, Walter E. Meyer, F. Danie Auret \\ Department of Physics, University of Pretoria, Private bag X20, 0028 Hatfield, South Africa
}

\begin{abstract}
Primary defects were introduced into $n$-type gallium nitride $(\mathrm{GaN})$ thin films by $1.6 \mathrm{MeV}$-proton bombardment at $20 \mathrm{~K}$. The electronic states of these defects were investigated by means of optical space charge spectroscopy. An up to now unreported primary defect, HP1, with an electronic state close to the valence band edge was detected. HP1 can be photo-ionised with photon energies of $3.4 \mathrm{eV}$. It was found to be stable up to $235 \mathrm{~K}$ but anneals quickly at temperatures above $240 \mathrm{~K}$.
\end{abstract}

Keywords: gallium nitride, GaN, primary defect, annealing, space charge spectroscopy, DLTS, photo-capacitance, optical capacitance voltage measurements

\section{Introduction}

Lattice defects in semiconductor crystals often introduce electronic states in the bandgap [1]. These states in turn affect the electronic properties of the material such as conduction type, conductivity and optical absorption. Primary lattice defects, e.g. vacancies and interstitials, can form if the material is exposed to high-energy particle radiation or during ion-implantations. Of course these primary defects are not nescessarily stable up to room-temperature but can become mobile and annihilate or react to more complicated defect complexes. The radiation hardness of a semiconductor material is therefore not only determined by the displacement energies of the host atoms. The material can appear to be highly radiation-tolerant because the induced primary lattice defects anneal even below room-temperature.

Space charge spectroscopic experiments such as deep-level transient spectroscopy or photo-capacitance (PCap) are known to be powerful tools for the investigation of electronic defect states in low concentrations. The experiments have in common that the capacitance of a space charge region provided by either a Schottky contact or a $p n$-junction is measured [2]. Thereby ionisation or neutralisation rates of these states manifest in changes of the capacitance (transients) from which the concentration of defects and defect parameters like activation enthalpies can be obtained.

Email address: schmidt.matthias@matschmi.de (Matthias Schmidt)

Preprint submitted to Physica B
Gallium nitride $(\mathrm{GaN})$ is a wide bandgap semiconductor, $E_{\mathrm{g}} \approx 3.4 \mathrm{eV}$ [3], with a remarkable radiationtolerance [4]. On the one hand this is due to the stiff lattice with high atomic displacement energies of $19 \mathrm{eV}$ and $22 \mathrm{eV}$ for gallium and nitrogen, respectively [5, 6]. On the other hand the material might occur to be radiation hard because of the low annealing temperatures of the radiation-induced primary lattice defects. This issue is still subject to investigation since so far most thermal annealing studies of radiation-induced defects were carried out above room-temperature where annealing processes cannot be excluded [7, 8]. Low-temperature annealing of electron irradiation-induced defects in the Ga-sublattice was observed by low-temperature optical detected electron paramagnetic resonance and photoluminescense studies [9]. The authors reported on annealing at temperatures as low as $60 \mathrm{~K}$.

In our previous work [10] we introduced lattice defects into $\mathrm{GaN}$ by low-temperature proton-bombardment of the sample. The characterisation of the defects, in particular their electronic states, was carried out in-situ by means of optical space charge spectroscopy $[2,11]$. These experiments allow to freeze the introduced primary defects such that annihilation or defect reactions do not take place. Furthermore the optical excitation allows for photo-ionisation of the electronic defect states and thus the whole bandgap can be scanned for defects at any temperature. Slowly increasing the temperature and simultaneously monitoring the sample capacitance enabled us to investigate annealing processes of primary defects. Amongst others the annealing of an acceptor-

September 5, 2013 
like state was observed in capacitance-temperature measurements with additional optical excitation. In this work we set out to investigate introduction and annealing of this defect more detailed.

\section{Experimental}

Samples and setup. A silicon doped $n-\mathrm{GaN}$ thin film was grown onto a 2-inch $a$-plane sapphire substrate by metal-organic chemical vapour deposition by AIXTRON SE Aachen, Germany. The film thickness was determined to $3.4 \mu \mathrm{m}$ by spectral optical transmission measurements. The wafer was cut into pieces in order to obtain a set of similar samples. In preparation of space charge spectroscopic measurements ohmic contacts made from a thermally annealed $\mathrm{Ti} / \mathrm{Al} / \mathrm{Ni} / \mathrm{Au}$ structure [12] as well as $15 \mathrm{~nm}$-thick semitransparent Pd Schottky contacts were deposited onto the samples. Thin gold wires were bonded to the contacts using conductive epoxy. The samples were mounted on the coolfinger of a Helium cryostat which was aligned to the beam-line of a van de Graaff accelerator. The capacitance of the samples was measured by an Agilent 4284A LCR metre. The monochromatic optical excitation was realised by a $1000 \mathrm{~W}-\mathrm{Xe} / \mathrm{Hg}$ arc lamp and a $1 / 4 \mathrm{~m}$ Cornerstone 260 grating monochromator. Optical fibres were used to pass the light from the monochromator into the vacuum chamber where it was focussed onto the sample. Details of the setup are published in $[10,13]$.

Defect studies. A pre-characterisation of the as-grown sample was carried out by capacitance-voltage $(C-V)$ measurements and by photo-capacitance spectroscopy (PCap). Using van Opdorp's method [14] the net doping density of the samples was determined to $1.7 \times$ $10^{17} \mathrm{~cm}^{-3}$. From PCap measurements (photon energies $3.0 \mathrm{eV}<h v<3.55 \mathrm{eV}$ ) the acceptor concentration was obtained to $1.3 \times 10^{16} \mathrm{~cm}^{-3}$.

In the first experiment the procedure that we proposed in [10] was used: (1) Firstly, the as-grown sample was cooled to $20 \mathrm{~K}$. (2) Then it was bombarded with 1. $6 \mathrm{MeV}$-protons at a fluence of $10^{14} \mathrm{~cm}^{-2}$ such that primary defects were introduced into the lattice. Thereby the low temperature ensured that the introduced defects were immobile and thus unable to anneal or react with each other. (3) The temperature was slowly increased to $330 \mathrm{~K}$. In this step primary defects became mobile and annealing processes or defect reactions occurred. (4) Finally the sample was cooled a second time to $20 \mathrm{~K}$. During the cycle, except for the bombardment, the sample was reverse-biased at $4 \mathrm{~V}$ and the capacitance was measured in dependence of the temperature, see Fig. 1(a). Since in this study we focussed on the annealing of acceptor states, the sample was illuminated with $3.4 \mathrm{eV}$-photons during all the measurements in order to photo-ionise electronic states in the vicinity of the valence band edge. This is illustrated in Fig. 1(b). In the capacitance-temperature $(C-T)$ characteristics of the as-bombarded sample the capacitance dropped irreversebly in the temperature range of $240 \mathrm{~K}$ to $280 \mathrm{~K}$, see insert of Fig. 1(a). This can only be explained by the annealing of a primary acceptor state which shall in the following be labelled HP1.

PCap measurements were conducted on a second sample from the same GaN wafer in order to gain more information about HP1. Again the sample was cooled to $20 \mathrm{~K}$ and proton-bombarded under the same conditions. Then the temperature was increased to $222 \mathrm{~K}$. Once this temperature was reached the sample was illuminated with monochromatic light and the capacitance change was measured in dependence of the photon energy. The results of these PCap measurements are depicted in Fig. 2(a) (solid line). Afterwards the sample temperature was further increased to $235 \mathrm{~K}$ for approximately $10 \mathrm{~min}$ and then again decreased to $222 \mathrm{~K}$ where another PCap spectrum was recorded (Fig. 2(a), dashed line). The PCap spectra of the as-bombarded and the $235 \mathrm{~K}$-annealed sample differ only marginally. However, a further repetition of this experiment with an annealing temperature of $240 \mathrm{~K}$ resulted in significant changes in the PCap spectrum, Fig. 2(a) (dotted line). This is in accordance with the results of the $C-T$ measurements. The annealing rate of HP1 at $240 \mathrm{~K}$ is therefore high enough such that HP1 anneals within ten minutes.

In order to understand where the HP1 level was introduced into the sample, optical capacitance-voltage (OCV) measurements [11] were conducted on another proton-bombarded sample at $222 \mathrm{~K}$. From these measurements the depth-resolved concentration profile of an optically active defect can be determined. Thereby four capacitance-voltage measurements were conducted: one in the dark and one under $h v=3.4 \mathrm{eV}$ illumination on the as-bombarded sample (black lines in Fig. 2(b)) as well as after the sample was annealed at $245 \mathrm{~K}$ (grey lines in Fig. 2(b)). The difference in the doping profiles measured in the dark and under illumination is equal to the concentration profile of the photo-ionised (acceptor-like) defect states. In the asbombarded as well as in the annealed sample a concentration gradient of these defects towards the substrate was measured. However, the difference between the doping profiles measured in the dark and under $3.4 \mathrm{eV}$ illumination was larger in the as-bombarded sample 

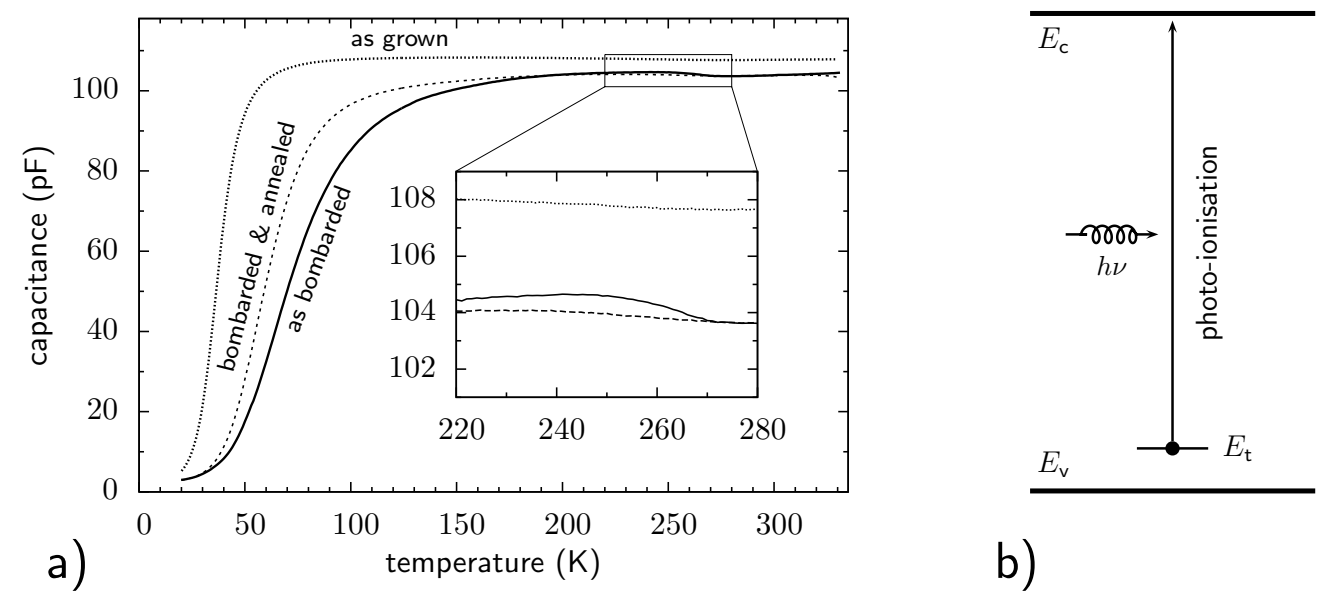

b)

Figure 1: a) Capacitance-temperature measurements during the proton-bombardment and defect annealing experiments. The sample was illuminated with monochromatic $3.4 \mathrm{eV}$-photons. In the inset a step in the capacitance caused by the annealing of the primary acceptor state HP1 is magnified. Data is taken from [10]. b) Photo-ionisation of an electronic state close to the valence band.
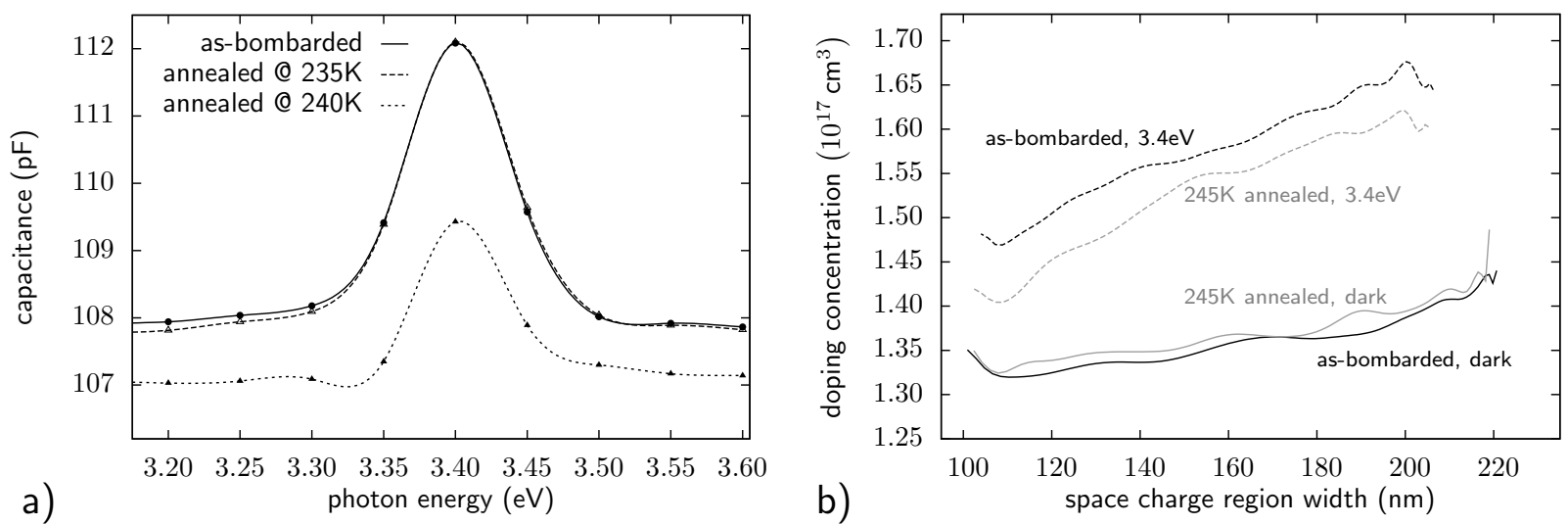

Figure 2: a) Photo-capacitance spectra recorded at $222 \mathrm{~K}$ (i) directly after the $\mathrm{p}^{+}$-bombardment as well as after the sample was exposed to (ii) $235 \mathrm{~K}$ and (iii) $240 \mathrm{~K}$, respectively. During the measurements the sample was reverse-biased at $4 \mathrm{~V}$. b) Doping profiles measured at $222 \mathrm{~K}$ by optical capacitance-voltage spectroscopy directly after the proton bombardment (black lines) as well as after the bombarded sample was exposed to $245 \mathrm{~K}$ (grey lines). The difference in the doping concentration measured in the dark (solid lines) and under $3.4 \mathrm{eV}$ illumination is the concentration of photo-ionised acceptors. 
than in the annealed sample. Therefore, the concentration of photo-ionised defect states in the as-bombarded sample is higher than after the sample was exposed to $245 \mathrm{~K}$. This confirms that an optically active defect has annealed. Together with the $C-T$ and the PCap measurements it is clear that this is the HP1 defect. From the difference in the doping profiles measured under illumination on the as-bombarded and the annealed sample the concentration of HP1 was found to be homogeneous in the space charge region and amounted to $(6 \pm 2) \times 10^{15} \mathrm{~cm}^{-3}$.

\section{Conclusion}

$\mathrm{GaN}$ thin film samples were $1.6 \mathrm{MeV}$-protonbombarded at $20 \mathrm{~K}$. This resulted in the introduction of an acceptor-like primary defect HP1. Whereas the total acceptor concentration exhibited a concentration gradient from the surface towards the substrate, the concentration of HP1 was homogeneous in the investigated region within the error bars of the experiment and amounted to $(6 \pm 2) \times 10^{15} \mathrm{~cm}^{-3}$. HP1 can be photoionised with photon-energies of approximately $3.4 \mathrm{eV}$. The defect is stable up to approximately $235 \mathrm{~K}$ and anneals quickly when the sample is exposed to temperatures above $240 \mathrm{~K}$.

\section{Acknowledgements}

The authors wish to thank AIXTRON SE (Aachen, Germany), in particular Dirk Fahle, Hannes Behmenburg, Christian Czekalla and Michael Heuken for providing the $\mathrm{GaN}$ thin film sample. We are grateful to the mechanical workshop of the Department of Physics at the University of Pretoria for the fabrication of numerous parts of the setup. Matthias Schmidt was funded by the Postdoctoral Fellowship Program of the University of Pretoria. This work is based upon research supported by the National Research Foundation (NRF). Any opinion, findings and conclusions or recommendations expressed in this material are those of the author(s) and therefore the NRF does not accept any liability in regard thereto.

[1] M. Lannoo and J. Bourgoin. Point defects in Semiconductors, Volume 1. Springer Verlag, Berlin, 1st edition, 1981.

[2] C.T. Sah, L. Forbes, L.L. Rosier and A.F. Tasch, Jr. Solid-State Electron. 131970 pp. 759-788

[3] H. Morkoc. Handbook of Nitride Semiconductor Devices, Volume 1. Wiley-VCH Verlag GmbH \& Co. KG, Weinheim, 1st edition, 2008.

[4] A.Y. Polyakov, S.J. Pearton, P. Frenzen, F. Ren, L. Liu and J. Kim. J. Mater. Chem. C 1 (2013)
[5] A. Ionascut-Nedelcescu, C. Carlone, A. Houdayer, H.J. von Bardeleben, J.-L. Cantin, and S. Raymond. IEEE T. Nucl. Sci., 49 (2002)

[6] J. Nord, K. Nordlund and J. Keinonen. Phys. Rev. B 68 (2003)

[7] S.A. Goodman, F.D. Auret, F.K. Koschnick, J.-M. Spaeth, B. Beaumont, P. Gibart Mater. Sci. Eng. B 71 (2000) pp. 100103

[8] A.Y. Polyakov, N.B. Smirnov, A.V. Govorkov, N.G. Kolin, D.I. Merkurisov, V.M. Boiko, A.V. Korulin and S.J. Pearton J. Vac. Sci. Technol. B 28 (2010) pp. 608-612

[9] K.H. Chow, L.S. Vlasenko, P. Johannesen, C. Bozdog, G.D. Watkins, A. Usui, H. Sunakawa, C. Sasaoka and M. Mizuta Phys. Rev. B 69 (2004) pp. 045207

[10] M. Schmidt, H. de Meyer, J. Janse van Rensburg, W.E. Meyer and F.D. Auret. Phys. Stat. Sol. B (2013) accepted, DOI: 10.1002/pssb.201349191

[11] M. Schmidt, H. v. Wenckstern, R. Pickenhain, M. Grundmann. Solid-State Electron. 75 (2012) pp.48-54

[12] J. Janse van Rensburg, F.D. Auret, V.S. Matias and A. Vantomme. Physica B 404 pp. $4411-4414$ (2009)

[13] M. Schmidt, J. Janse van Rensburg, H. de Meyer , W.E. Meyer, F.D. Auret, F. Schmidt, H. von Wenckstern. in Proceedings of SAIP2012, the 57th Annual Conference of the South African Institute of Physics, under review. Available online at http: \\www.saip.org.za

[14] C. van Opdorp. Solid-State Electron. 11 pp. 397-406 (1968) 